\title{
Meso-scale analysis of deformation wave heating in metalized solid explosive
}

\author{
S. Chakravarthy ${ }^{1, \mathrm{a}}$, K.A. Gonthier ${ }^{1, \mathrm{~b}}$, and C. Rumchik ${ }^{2, \mathrm{c}}$ \\ 1 Mechanical Engineering Department, Louisiana State University, Baton Rouge, LA 70803, USA \\ 2 U.S. Air Force Research Laboratory, AFRL-RWME, Eglin AFB, FL 32542, USA
}

\begin{abstract}
Deformation induced heating of reactive solids is a physically complex process. As such, the effects of meso-structure, component thermomechanical properties, component mass fractions, and porosity on their impact response is not well-understood. In this study, an explicit, 2-D, Lagrangian finite and discrete element technique is used to examine thermomechanical fields in metal-explosive (aluminum-HMX) particle mixtures due to piston supported uniaxial deformation waves. The meso-scale description uses a plane strain, thermoelastic-viscoplastic and friction constitutive theory to describe the motion and deformation of individual particles, and an energy consistent, penalty based method to describe inter-particle contact. The deformation response of material having an initial solid volume fraction of $\phi_{s}^{0}=0.835$ is characterized for different metal mass fractions and wave strengths. Predictions indicate that the response can be classified into strength dominated and pressure dominated regions depending on wave strength. Average thermomechanical fields that define the macro-scale wave structure are found to differ both qualitatively and quantitatively between the two regions.
\end{abstract}

\section{Introduction}

Reactive solids often consist of mixtures of high-explosive, metal, and oxidizer particles (size $\approx$ 10-200 $\mu \mathrm{m}$ ) embedded within a polymeric binder. It has been shown that inclusion of metal in explosive mixtures can affect their impact sensitivity, detonation, and post-detonation behavior, though it remains fundamentally unclear how meso-structure, component (or phase) thermomechanical properties, and metal mass fraction, affect the impact sensitivity of the mixture. The objectives of this study are two-fold: 1) to characterize the relative importance of phase-specific volumetric and surface dissipation and its dependence on meso-structure, metal mass fraction, and wave strength; and, 2) to investigate macro-scale manifestations of the particle-scale response by spatially averaging mesoscale fields over suitably chosen representative volumes (areas). The first objective is important for identifying physical mechanisms responsible for ignition, whereas the second objective is important for developing macro-scale, multiphase constitutive theories to accurately assess the performance of reactive solids in practice $[1,2]$. Multiphase theories are routinely posed in terms of phase interaction terms that implicitly account for particle-scale phenomena that are difficult to experimentally resolve.

The initial material configuration used in this study consists of circular, randomly packed aluminum and HMX particles having an average diameter of $\bar{d} \approx 60 \mu \mathrm{m}$, as illustrated in Fig. 1. Interparticle voids are massless. This idealized geometry is chosen to mitigate singularities that would occur

\footnotetext{
a e-mail: schakr4@lsu.edu

b e-mail: gonthier@me.1su. edu. Corresponding author.

c e-mail: chad.rumchik@eglin.af.mil
}

This is an Open Access article distributed under the terms of the Creative Commons Attribution-Noncommercial License 3.0, which permits unrestricted use, distribution, and reproduction in any noncommercial medium, provided the original work is properly cited. 


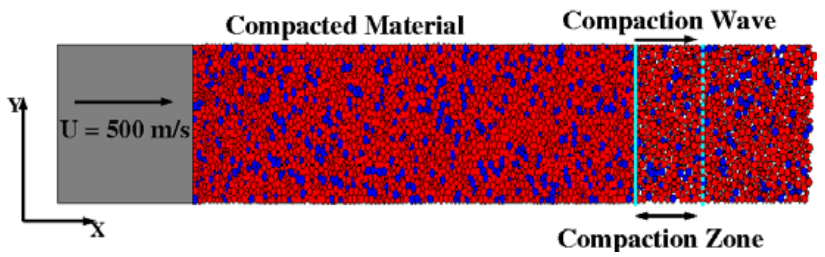

Fig. 1. Illustration of an aluminized HMX mixture corresponding to $\lambda_{m}=0.2$ and $U_{p}=500 \mathrm{~m} / \mathrm{s}$. The blue particles are metal and the red particles are HMX.

in the vicinity of sharp particle corners that are impossible to numerically resolve. Nonetheless, this simplified initial configuration still enables leading-order effects of viscoplastic deformation and interparticle friction to be assessed. The average initial solid volume fraction of the material is $\phi_{s}^{0}=0.835$. For brevity, only predictions for piston speeds of $U_{p}=100$ and $500 \mathrm{~m} / \mathrm{s}$ are discussed as they enable differences between the strength and pressure dominated deformation regions to be highlighted.

\section{Model description}

The model problem is posed as a multi-particle contact problem for the evolution of thermomechanical fields within particles and interactions between particles. Evolution equations for the momentum and energy of each particle are given in Lagrangian form by the following:

$$
\begin{gathered}
\rho \ddot{\mathbf{u}}=\boldsymbol{\nabla} \cdot \boldsymbol{\sigma}, \\
\rho c_{v} \dot{T}=-\nabla \cdot \mathbf{q}+\rho r .
\end{gathered}
$$

Here, $\rho$ is the local density, $\sigma$ is the Cauchy stress tensor, $r$ is the deformation induced heating, $\mathbf{q}$ is the heat flux, $c_{v}$ is the constant volume specific heat, and $\boldsymbol{\nabla} \equiv \frac{\partial(\bullet)}{\partial \mathbf{x}}$ is the spatial gradient operator. Particles are assumed to be initially stationary and stress free. To satisfy the entropy inequality, a Clausius Duhem entropy constraint is used which is given by:

$$
\rho \dot{\eta}-T^{-1}(\rho r-\boldsymbol{\nabla} \cdot \mathbf{q})+\mathbf{q} \cdot \boldsymbol{\nabla} T^{-1} \geq 0,
$$

where $\eta$ is the mass-specific entropy. Additionally, the following contact conditions are imposed on particle boundaries $\Gamma$ :

$$
\begin{gathered}
\boldsymbol{\sigma} \cdot \mathbf{n}=\mathbf{t}_{c} \text { on } \Gamma \forall \mathrm{t}, \\
-k \boldsymbol{\nabla} T \cdot \mathbf{n}=q_{f}+q_{c} \text { on } \Gamma \forall \mathrm{t} .
\end{gathered}
$$

Here, $\mathbf{n}$ is the local unit normal vector to $\Gamma, \mathbf{t}_{c}$ is the local contact traction vector, $q_{f}$ is local frictional heat flux, $q_{c}$ is the local heat flux needed to impose ideal thermal contact, and $k$ is the thermal conductivity. The stress-strain behavior of the particles is modeled by a hyperelastic, multiplicative, finite strain constitutive model with a Von-Mises yield criterion and viscoplastic flow rule, and friction is modeled using an Amontons-Coulomb stick/slip theory. A detailed description of the model equations, contact conditions, and constitutive theory is given in Ref. [3]. Key thermomechanical properties for HMX and aluminum used in the simulations are given in Table 1.

\subsection{Numerical technique}

The model equations are computationally solved subject to the boundary conditions using a combined finite and discrete element technique. The finite-element method (FEM) is used to numerically integrate the unsteady 2-D conservation equations and the viscoplastic flow rule governing inelastic 
Table 1. Thermomechanical properties.

\begin{tabular}{lll}
\hline Property & HMX & Aluminum \\
\hline Density $\left(\mathrm{kg} / \mathrm{m}^{3}\right)$ & 1903 & 2400 \\
Yield Stress $(\mathrm{GPa})$ & 0.37 & 0.25 \\
Poisson's Ratio (-) & 0.20 & 0.35 \\
Young's Modulus $(\mathrm{GPa})$ & 24.0 & 70.0 \\
Bulk Modulus $(\mathrm{GPa})$ & 13.33 & 77.8 \\
Specific Heat $(\mathrm{kJ} / \mathrm{kg} / \mathrm{K})$ & 1.50 & 0.90 \\
Thermal Conductivity $(\mathrm{W} / \mathrm{m} / \mathrm{K})$ & 0.5 & 237.0 \\
Longitudinal Sound $\mathrm{speed}(\mathrm{km} / \mathrm{s})$ & 2.65 & 5.69 \\
Shear Sound Speed $(\mathrm{km} / \mathrm{s})$ & 2.29 & 3.29 \\
\hline
\end{tabular}

deformation, whereas the discrete element method (DEM) is used to account for interactions between particles. The DEM utilizes a conservative potential based penalty method in which the normal contact traction between particles is estimated by penalizing their penetration, and frictional tractions are estimated using a penalty regularized Amontons-Coulomb law [3]. The initial particle configuration is generated using a pseudo-gravity drop method that consists of two steps. First, an initial packing arrangement is generated by randomly placing particles within the domain so that their centers lie outside the volume encompassed by other particles. Then, forces arising due to particle overlap drives rearrangement of particle positions in such a way as to eliminate overlap while continuously settling under the influence of a gravitational force [3]. Particles are discretized using constant strain triangular elements, where each particle contains approximately 400-800 finite elements for all simulations performed in this study, depending on its size. The combined FEM/DEM algorithm has been verified, and is nominally second-order accurate in both space and time.

\section{Predictions}

Preliminary predictions are given in this section for piston supported uniaxial deformation waves in aluminized HMX having $\phi_{s}^{0}=0.835$. Macro-scale wave profiles are obtained by computing spatial transverse averages over meso-scale fields using an averaging width of $200 \mu \mathrm{m}$. Though computed average profiles do vary with averaging width, numerical experiments indicate that meaningful results are obtained provided that this width is smaller than approximately half the size of the macro-scale wave thickness.

Figure 2 gives predicted variations in the Hugoniot curves with metal mass fraction $\lambda_{m}$. Figures 2(a) and (b) express these curves in the $U_{p}-P_{m i x}$ and $U_{p}-D$ planes, respectively, where $P_{m i x}$ is the average equilibrium mixture pressure behind the wave and $D$ is the corresponding steady wave speed. To this end, the axial dimension of the computational domain was chosen sufficiently long to guarantee that steady waves evolved. Here, we consider piston speeds within the range $50 \leq U_{p} \leq$ $500 \mathrm{~m} / \mathrm{s}$ and metal mass fractions within the range $0 \leq \lambda_{m} \leq 1$, where piston speeds in excess of approximately $U_{p} \geq \hat{U}_{p}=250 \mathrm{~m} / \mathrm{s}$ result in nearly complete material consolidation for all values of $\lambda_{m}$. This critical value of piston speed separates two well-defined impact regions. We refer to the lower pressure region corresponding to $U_{p}<\hat{U}_{p}$ as strength dominated because material strength is important in preventing complete pore collapse. We refer to the higher pressure region corresponding to $U_{p} \geq \hat{U}_{p}$ as pressure dominated because the resulting pressures are sufficient to completely eliminate porosity. Material strength plays a less significant role than pressure in the pressure dominated region.

A few observations are noteworthy. First, $P_{m i x}$ monotonically increases with $\lambda_{m}$ due to the high acoustic impedance of aluminum which causes the reflection of acoustic energy at explosive-metal interfaces thereby enhancing the deformation and pressure of explosive particles. This observation is commensurate with data for neat granular $\operatorname{HMX}\left(\lambda_{m}=0\right)$ and neat porous aluminum $\left(\lambda_{m}=1\right)$ [4]. Here, neat material refers to one that consists of only a single or pure-phase component. Second, 


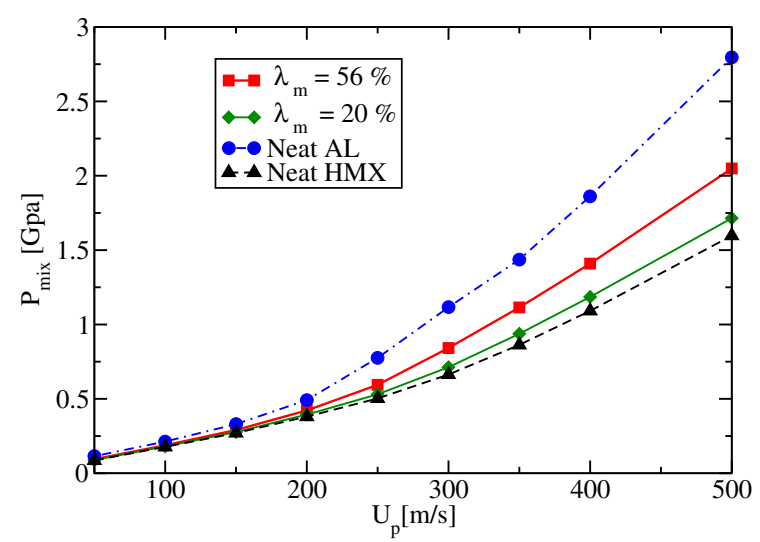

(a)

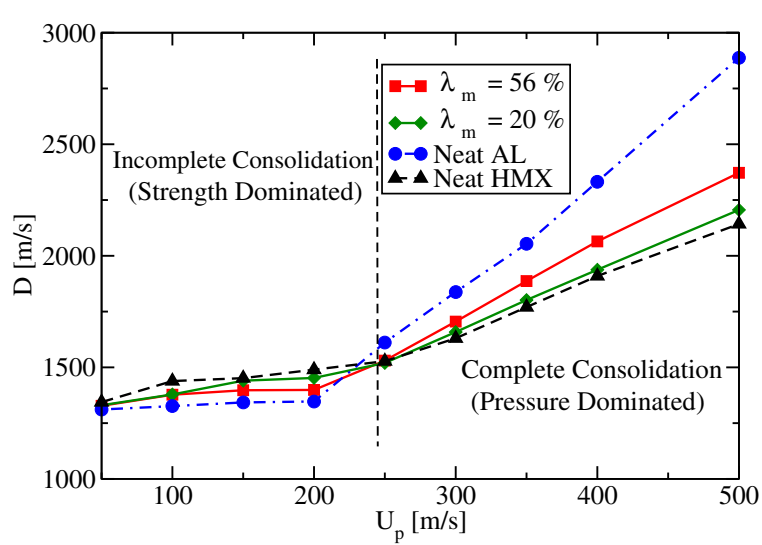

(b)

Fig. 2. Predicted Hugoniot curves for a material having $\phi_{s}^{0}=0.835$ and metal mass fraction $\lambda_{m}$ : (a) the $P-U_{p}$ plane; (b) the $D-U_{p}$ plane. Here, neat refers to a single phase granular material.

variations in $D$ with $\lambda_{m}$ qualitatively differ between the two impact regions. In the strength dominated region, $D$ slightly decreases with increasing $\lambda_{m}$ whereas the opposite trend is predicted for the pressure dominated region. Though speculative at this juncture, this prediction is plausible because the lower aluminum strength enhances dissipation by plastic work during pore collapse, even for neat granular aluminum $\left(\lambda_{m}=1\right)$, resulting in slightly lower wave speeds than those associated with neat HMX in the strength dominated region. However, dissipation by plastic flow becomes more geometrically constrained within wave profiles in the pressure dominated region due to the elimination of porosity resulting in higher wave speeds, as anticipated. Though not shown here for brevity, the weak variation in $D$ with $U_{p}$ in the strength dominated region is largely due to inter-particle friction during pore collapse which enhances the rigidity of the material; as such, we also refer to this region as being frictionally rigid. Simulations performed for frictionless contact indicate a larger decrease in $D$ as $U_{p} \rightarrow 0$ in the strength dominated region.

We now briefly focus on predictions for wave energetics. Figures 3(a) and (b) give spatial variations in average phase-specific plastic heating rate $\dot{W}_{p}$ within wave profiles corresponding to $U_{p}=$ $100 \mathrm{~m} / \mathrm{s}$ and $U_{p}=500 \mathrm{~m} / \mathrm{s}$, respectively, for a material having $\lambda_{m}=0.2$. Here, the average phasespecific heating rate and its standard deviation are computed based on the local phase-specific mass affected by plastic work. Also shown in these plots for comparison is the equivalent predicted profile for neat HMX having the identical initial configuration as the aluminized HMX mixture. Neat HMX forms a proper baseline for establishing the relative impact sensitivity of aluminized HMX. Average 


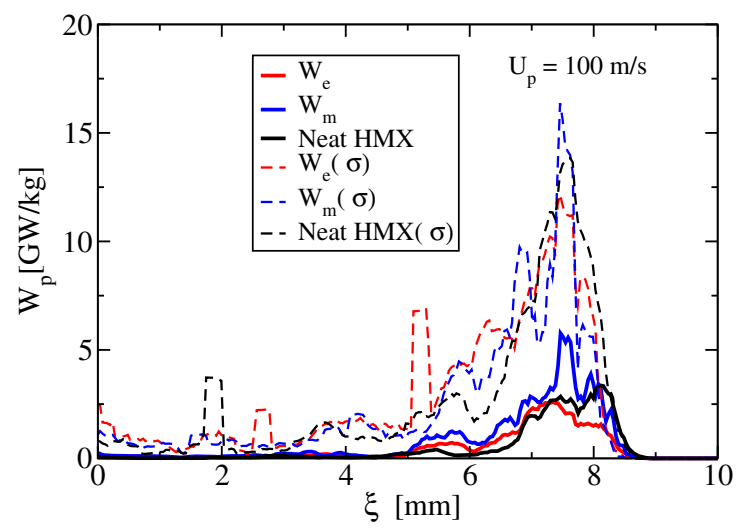

(a)

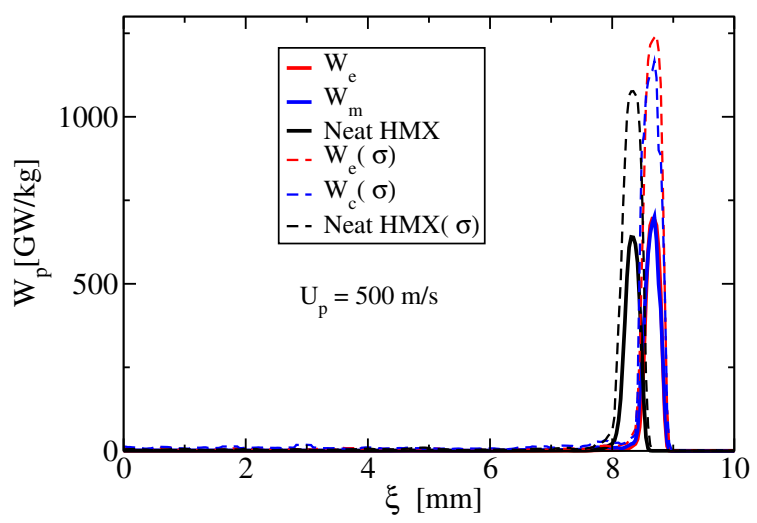

(b)

Fig. 3. Predicted average plastic heating rate $\dot{W}_{p}$ profiles for a material having $\phi_{s}^{0}=0.835$ and $\lambda_{m}=0.2$ : (a) $U_{p}=100 \mathrm{~m} / \mathrm{s}$; (b) $U_{p}=500 \mathrm{~m} / \mathrm{s}$. Corresponding standard deviations are given by dashed lines.

heating rates within wave profiles are important to analyze because they can be directly compared to predictions given by macro-scale multiphase theories and because they give a good indication of deformation wave thickness.

As seen in Fig. 3(a) for $U_{p}=100 \mathrm{~m} / \mathrm{s}$, the metal plastic heating rate exceeds that of the explosive within the wave profile for aluminized HMX. Further, the explosive plastic heating rate is marginally lower than that of the equivalent neat HMX configuration. This prediction is consistent with our previous assertion for strength dominated waves for which the inclusion of aluminum reduces wave speed by enhancing plastic dissipation within aluminum particles during pore collapse, thereby reducing explosive heating relative to neat HMX. Standard deviations about the average rates are large due to localized plastic deformation near inter-particle contacts. The predicted deformation wave thickness is approximately $4 \mathrm{~mm}$.

Figure 3(b) gives predictions for $U_{p}=500 \mathrm{~m} / \mathrm{s}$. This case, which corresponds to a pressure dominated wave, results in a marginally higher explosive plastic heating rate than that of neat HMX, again possibly due to enhanced geometrical confinement associated with the elimination of porosity. The predicted deformation wave thickness is approximately $1 \mathrm{~mm}$. The implications on impact sensitivity of these marginal differences in explosive heating relative to that of neat HMX remain an ongoing topic of our research.

Figure 4 gives corresponding spatial variations in average phase-specific friction heating rate $\dot{W}_{f}$ within wave profiles. A friction coefficient of $\mu=0.25$ was used for all simulations performed in this work because parametric studies indicate that this value results in maximum frictional dissipation. 


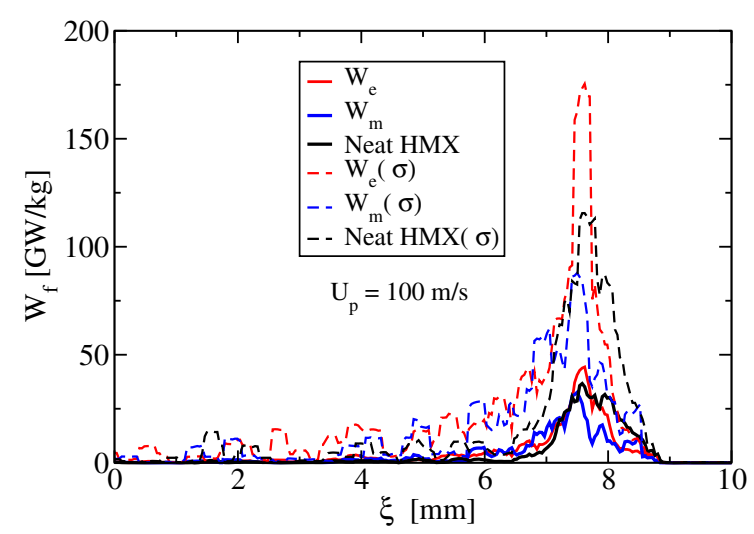

(a)

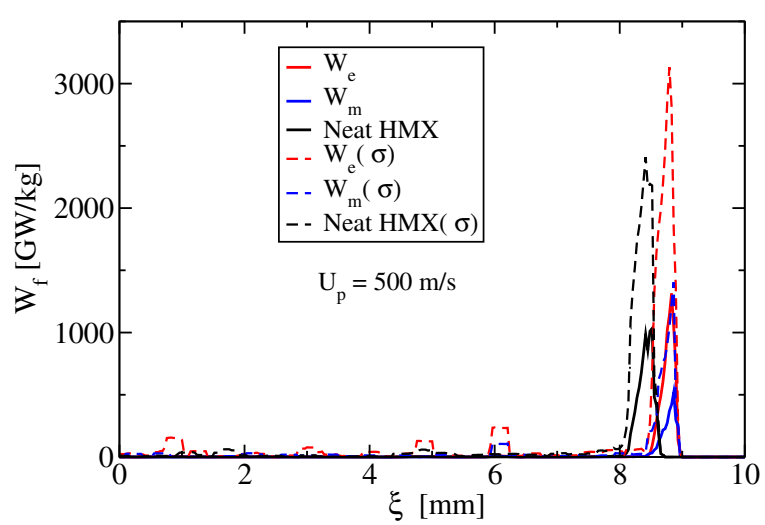

(b)

Fig. 4. Predicted average frictional heating rate $\dot{W}_{f}$ profiles for a material having $\phi_{s}^{0}=0.835$ and $\lambda_{m}=0.2$ : (a) $U_{p}=100 \mathrm{~m} / \mathrm{s}$; (b) $U_{p}=500 \mathrm{~m} / \mathrm{s}$. Corresponding standard deviations are given by dashed lines.

Here, the average phase-specific heating rate and its standard deviation are computed based on the local phase-specific mass affected by friction work, which is approximated by finite-element mass within the averaging area experiencing sliding contact. Based on this approximation, friction heating rates exceed plastic heating rates in all cases as friction work is a surface phenomenon. Though plastic work affects a larger mass of material, friction work remains a significant source of dissipation for pressure dominated waves. Importantly, friction work is believed to be a primary mechanism for the formation of hot-spots that are responsible for ignition of reactive solids.

\section{Conclusion}

A 2-D, Lagrangian, combined discrete and finite element technique was used to computationally characterize piston supported uniaxial waves in granular metal-explosive (aluminum-HMX) mixtures. Predictions indicate that the impact response can be divided into material strength and pressure dominated regions, depending on piston speed. Strength dominated, low pressure waves result in incomplete material consolidation, whereas pressure dominated waves are sufficiently strong to eliminate porosity. Predicted Hugoniots indicate that, for a material having $\phi_{s}^{0}=0.835$, the wave speed slightly decreases with increasing metal mass for strength dominated waves corresponding to $U_{p}<\hat{U}_{p}=250 \mathrm{~m} / \mathrm{s}$, whereas the opposite trend is predicted for pressure dominated waves corresponding to $U_{p} \geq \hat{U}_{p}$. Inclusion of metal is found to marginally decrease the average explosive heating rate within strength 
dominated waves, and to increase explosive heating rate within pressure dominated waves. Friction work is shown to be a potentially significant hot-spot formation mechanism, even for pressure dominated waves. The average equilibrium pressure behind waves was found to increase with metal mass for both impact regions due to the high acoustic impedance of the metal. It is emphasized that careful interpretation of plastic and frictional heating rates, and meso-scale thermomechanical fields, is necessary to properly characterize the thermal response of reactive solids.

This work was supported by the U.S. Air Force Research Laboratory, Munitions Directorate, AFRL-RWME, Eglin AFB, Florida, USA. 96ABW-2009-0327.

\section{References}

1. Gonthier, K.A., Journal of Applied Physics 95(7), (2004) pp. 3482-3494.

2. Gonthier, K.A., and Rumchik, C.G., Technical Report ME-TSI-09, Mechanical Engineering Department, Louisiana State University, Baton Rouge, Louisiana, USA, 2009.

3. Panchadhar, R., and Gonthier, K.A., Computational Mechanics 44, (2009) pp. 717-744.

4. Marsh, S.P., LASL Shock Hugoniot Data (University of California Press, Berkeley, CA, USA, 1980. ISBN-0-520-04008-2). 